\title{
Challenging Topics in Neuroanesthesia and Neurocritical Care
}

\author{
Zahid Hussain Khan (Editor). Springer, 2017, 339 pages. ISBN 978-3-319-41443-0
}

\author{
Daniel Chartrand, MD, PhD, FRCPC (D)
}

Received: 15 February 2018/Accepted: 16 February 2018/Published online: 1 March 2018

(c) Canadian Anesthesiologists' Society 2018

The textbook, Challenging Topics in Neuroanesthesia and Neurocritical Care, is the result of an international collaboration of authors from ten countries. It covers three main areas, with the first part emphasising traumatic brain and spinal cord injury, the second dealing with airway and pain management, and the third covering 16 other various topics in neuroanesthesia and neurocritical care.

The intended target audience appears to be the anesthesia and/or critical care resident who is doing a first rotation in neuroanesthesia or neurocritical care. Some basic concepts are covered at the junior resident level, whereas others will interest even seasoned neuroanesthesiologists. Accordingly, although this book describes the standard management of "routine" cases, it also includes expert "tips" and "tricks" applicable to some potentially difficult situations and dangerous conditions.

In the first section of the book, Chapter 1 presents the key concepts of intracranial compliance and traumatic brain injury (TBI) management, followed by a detailed discussion of brain tissue oxygen pressure monitoring in Chapter 2. The predictors of outcomes after a TBI are covered in Chapter 3, with real-life case scenarios described in Chapter 4 to help the reader understand the process of declaring brain death. Chapter 5 is devoted to a poorly understood phenomenon - paroxysmal sympathetic hyperactivity - which follows diverse neurological injuries. The 13 pages of Chapter 6 briefly present the basics of the pathophysiology, classification, and management of acute

D. Chartrand, MD, PhD, FRCPC ( $\square)$

Department of Anesthesia, McGill University, Montreal, QC, Canada

e-mail: daniel.chartrand@mcgill.ca spinal cord injury. This part of the book is completed by a chapter covering aneurysmal subarachnoid hemorrhage, a life-threatening pathology that includes complex derangements of neurologic, cardiac, pulmonary, and endocrine function.

The second part of Challenging Topics in Neuroanesthesia and Neurocritical Care starts with Chapter 8, which briefly covers airway management for general neurosurgical procedures as well as for some very specific procedures (e.g., awake craniotomy, pituitary surgery, cervical spine surgery). This material can certainly be used to initiate discussions during a clinical rotation. In addition, it provides 67 references, offering more reading to complete the learning process. In Chapter 9, the editor presents his own airway classification for edentulous patients proposes the "Upper Lip Catch Test" that, in my opinion, needs more thorough assessment. Airway management during neurocritical care is discussed in Chapter 10, which explains the general principles at a level easily grasped by medical students and possibly by the neurocritical care resident with a background in general neurology or internal medicine. Airway management is briefly explained, and practical tricks are offered to address specific situations such as trauma, stroke, and status epilepticus. The second part of this textbook ends with a short chapter on postoperative pain management after craniotomy.

The 16 chapters of the third part of Challenging Topics are devoted to a variety of neuroanesthesia and neurocritical care topics. For example, a very short chapter on anesthesia for awake craniotomy concentrates on local anesthesia and its toxicity. However, because awake craniotomy also requires extensive knowledge of the underlying neuroanesthesia principles, as well as advanced airway management and skillful management of systemic and cerebral hemodynamics, the reader will 
need to refer to other parts of this book and other documents for a thorough understanding of this topic. Another chapter is devoted to total intravenous anesthesia techniques for neurosurgery, although, once again, the reader will need to at least peruse some of the suggested references to gain sufficient knowledge of this important area.

Specific chapters provide a brief introduction to some potential neuroanesthesia challenges, including pediatric craniofacial procedures and airway problems in acromegalic patients. Chapter 16, for instance, focuses on airway and fluid management of patients in a prone position, providing good explanations and many expert tips. Chapter 17 nicely summarizes the numerous anesthetic considerations involved in posterior fossa surgeries, and Chapter 18 does the same for anesthesia for pituitary surgery.

More short chapters follow that cover venous air embolism (Chapter 19), blood glucose management (Chapter 20), anesthesia for deep brain stimulation (Chapter 21), and intraoperative magnetic resonance imaging (Chapter 22). They are followed by a comprehensive chapter on sedation in the neurocritical intensive care unit, which also introduces the emerging trend of inhalation sedation with volatile anesthetics.

Chapter 24 constitutes a good introduction to transcranial Doppler and duplex ultrasonography. In
Chapter 25, the editor discusses postoperative intracranial hemorrhage, a potentially devastating complication, although referring only to his own interventional study on 37 patients where he suggests that occult bleeding during primary hemostasis can be identified by inducing hyperdynamic stress with ketamine or hypervolemia. This may be a novel approach, but further studies are required before its potential benefits and risks are more fully understood. Finally, the challenging topics of neuroprotection and sepsis are briefly discussed in Chapters 26 and 27.

In summary, trainees starting a rotation in neuroanesthesia or neurocritical care could certainly use this book as an introduction to the many challenging situations they will be confronting. Further reading of some key references and discussion with the attending staff, however, will still be required. Finally, clinicians who are only occasionally challenged by neuroanesthesia cases or neurocritical care will also find the information in Challenging Topics in Neuroanesthesia and Neurocritical Care useful.

Conflicts of interest None declared.

Editorial responsibility This submission was handled by Dr. Hilary P. Grocott, Editor-in-Chief, Canadian Journal of Anesthesia. 